\title{
O CUIDADO DO PACIENTE ONCOLÓGICO COM DOR CRÔNICA NA ÓTICA DO ENFERMEIRO ${ }^{1}$
}

Lili Marlene Hofstätter da Silva ${ }^{2}$

Márcia Maria Fontão Zago ${ }^{3}$

Silva LMH, Zago MMF. O cuidado do paciente oncológico com dor crônica na ótica do enfermeiro. Rev Latino-am Enfermagem 2001 julho; 9(4):44-9.

O objetivo deste estudo foi identificar como o enfermeiro interpreta o cuidado com o paciente oncológico com dor crônica. O estudo foi desenvolvido com oito enfermeiros de uma instituição hospitalar. Os dados foram coletados por entrevistas semi-estruturadas e na análise identificamos três categorias analíticas: a avaliação da dor crônica do paciente oncológico, a importância do cuidado multidisciplinar e as dificuldades para o cuidado do paciente. Os resultados mostram que os enfermeiros têm dificuldades em desenvolver o cuidado com o paciente devido à falta de conhecimentos específicos sobre o câncer, dor crônica e sua terapêutica, como também, nas habilidades expressivas para promoverem o apoio psicológico adequado.

PALAVRAS CHAVE: dor, oncologia, cuidados de enfermagem, enfermeiros

\section{THE CARE TO CANCER PATIENTS WITH CHRONIC PAIN IN THE VIEW OF NURSES}

This study aims to understand how nurses interpret the care given to cancer patients with chronic pain. The informers were eight nurses from a public hospital. Data were collected on the basis of semi-structured interviews and the analysis identified three analytical categories: the evaluation of patients' pain, the importance of multidisciplinary care and nurses' difficulties to care for these patients. The results show that nurses have difficulties to care for such patients and need to update their specific knowledge related to cancer, chronic pain and its treatment as well as their skills to deal with emotions in order to promote adequate psychological support.

KEY WORDS: pain, oncology, nursing care, nurses

\section{EL CUIDADO DEL PACIENTE ONCOLÓGICO CON DOLOR CRÓNICO EN LA VISIÓN DEL ENFERMERO}

El objetivo de este estudio fue identificar como el enfermero interpreta el cuidado para el paciente oncológico con dolor crónico. El estudio fue desarrollado con ocho enfermeros de una institución hospitalaria. Los datos fueron recolectados por entrevistas semi-estructuradas y en el análisis identificamos tres categorías analíticas: la evaluación del dolor crónico del paciente oncológico, la importancia del cuidado multidisciplinario y las dificultades para el cuidado del paciente. Los resultados muestran que los enfermeros tienen dificultades para desarrollar el cuidado con el paciente debido a la falta de conocimientos específicos con respecto al cáncer, dolor crónico y su tratamiento, como también, en las habilidades expresivas para la promoción del apoyo psicológico adecuado.

PALABRAS CLAVES: dolor, oncología, cuidados de enfermería, enfermeros

\footnotetext{
${ }^{1}$ Este artigo é parte da Dissertação de Mestrado apresentada ao Programa de Enfermagem Fundamental da Escola de Enfermagem de Ribeirão Preto da Universidade de São Paulo em junho de 2000; ${ }^{2}$ Enfermeira, Professora Mestra do Departamento de Enfermagem da Universidade Estadual do Oeste do Paraná - UNIOESTE; ${ }^{3}$ Enfermeira, Professor Associado da Escola de Enfermagem de Ribeirão Preto da Universidade de São Paulo, Centro Colaborador da OMS para o desenvolvimento da pesquisa em enfermagem. Orientadora do estudo, e-mail: mmfzago@eerp.usp.br
} 
INTRODUÇÃO

$\boldsymbol{E}_{\text {ste estudo surgiu das reflexões da nossa prática }}$ profissional com o cuidado do paciente oncológico com dor crônica (PODC), quando o procedimento básico consistia apenas em aliviar a dor, administrando os analgésicos prescritos. Após a inserção na carreira universitária, essa preocupação ressurgiu ao acompanhar as atividades práticas de alunos do curso de graduação em uma instituição hospitalar governamental e assumir a responsabilidade por estimulá-los a desenvolver intervenções com qualidade.

Além da preocupação com a formação do aluno de enfermagem, observamos a atuação da equipe de enfermagem daquela instituição e questionamos sobre o cuidado com o PODC que nos parecia não suprir as necessidades do paciente.

Entretanto, nas últimas décadas o conhecimento, o conceito e as intervenções terapêuticas para a dor crônica do paciente oncológico tiveram uma grande evolução, porém, a capacitação do enfermeiro ainda é inadequada ${ }^{(1)}$.

A dor crônica é hoje conceituada como um fenômeno complexo e multifatorial, que envolve aspectos orgânicos e psicossociais. 0 tratamento neurofisiológico e neurofarmacológico são considerados eficazes mas a abordagem multidisciplinar, que surgiu na década de 80, a psico-oncologia, tem importância ímpar para a qualidade de vida do PODC. Essa abordagem emprega modalidades de intervenções fisiológicas, emocionais, cognitivas e sociais ${ }^{(2)}$. Independentes do enfoque a ser utilizado na assistência ao PODC, o enfermeiro deve destacar-se como profissional atualizado e capacitado para promover o cuidado deste paciente.

Assim, a finalidade deste estudo é buscar lacunas na assistência que possam direcionar o ensino do cuidado com o PODC, na graduação de enfermagem e na educação continuada dos enfermeiros. Para tanto, nosso objetivo é identificar como o enfermeiro interpreta o cuidado com o PODC.

\section{A DOR CRÔNICA NO PACIENTE ONCOLÓGICO E O CUIDADO DE ENFERMAGEM}

As descobertas mais importantes sobre a dor ocorreram após a II Guerra Mundial, decorrentes da expansão do conhecimento sobre anatomia e fisiologia, e a utilização do método científico para investigar o significado da dor ${ }^{(3)}$.

Em 1960, Melzack definiu dor como uma sensação e, em 1979, a Organização Mundial de Saúde (OMS) complementou essa definição ao considerar a dor como uma experiência sensorial e emocional desagradável, associada à lesões reais ou potenciais ou descritas em termos de tais lesões ${ }^{(4)}$. Essa definição é atualmente utilizada.
As pesquisas também demonstram que a percepção e a reação à dor variam entre indivíduos com uma mesma doença, de igual localização e extensão e podem levar a diferentes graus de sofrimento. Essas diferenças individuais dependem do sexo, raça, cultura e história do indivíduo.

Dentre os fatores que influenciam a sensação dolorosa, evidenciam-se os sentimentos e as experiências emocionais como mágoa, luto, temor, angústia e culpa. Portanto, a reação a um estímulo doloroso é individual, depende do estado físico e emocional do sujeito em situação a dor.

Assim, a dor é considerada como uma síndrome resultante da interpretação do aspecto físico-químico do estímulo nocivo e da sua interação com as características individuais como o humor, o significado simbólico atribuído ao fenômeno sensitivo e os aspectos culturais e afetivos dos indivíduos ${ }^{(1)}$.

Os pacientes podem experimentar dois tipos de dor: a dor aguda e a crônica. A dor crônica é definida quanto ao tempo de duração. Há autores que consideram a dor crônica como uma síndrome com duração de 3 a 6 meses ${ }^{(4-5)}$.

A dor crônica é freqüente em doentes com câncer e pode ser devido ao tumor primário ou suas metástases, à terapia anticancerosa (cirurgia, radioterapia ou quimioterapia) e aos métodos de diagnóstico. Em algumas situações, ela pode estar relacionada a causas psicossociais ${ }^{(4)}$.

A dor crônica, no paciente oncológico, incapacita-o e acarreta modificações danosas no âmbito orgânico, emocional, comportamental e social.

Um aspecto importante a ser considerado é que dor crônica acomete cerca de $50 \%$ dos pacientes com câncer em todos os estágios da doença e em $70 \%$ nas neoplasias avançadas ${ }^{(1)}$.

Atualmente, o mecanismo reconhecido como predominante da dor crônica do câncer é a invasão tumoral, com dano tecidual e ativação de neuroreceptores periféricos (receptores das sensações dolorosas). Os receptores da dor (nociceptores) são terminações nervosas, livres da pele, que respondem apenas a um estímulo intenso, potencialmente danoso. Esse estímulo pode ser de natureza mecânica, térmica e química ${ }^{(6)}$.

De qualquer modo, o relato da experiência dolorosa pelo paciente aos profissionais de saúde é fundamental para a compreensão do quadro álgico, implementação de medidas analgésicas e avaliação da eficácia terapêutica.

Se no tratamento de pacientes com dor crônica é necessário considerar vários fatores que interagem no processo, ressalta-se a importância de analisar e compreender a dor como decorrente desses fatores, e não isoladamente, visto que o objetivo do tratamento é a reabilitação global do indivíduo e não apenas corrigir um dos aspectos isolados de sua expressão sintomática ${ }^{(4)}$. Nesse sentido, a avaliação da dor, pelo profissional, é o ponto fundamental para o planejamento do tratamento e do cuidado. 
A preocupação com a compreensão da dor crônica do paciente oncológico tem aumentado muito nestes últimos anos, assim como os recursos terapêuticos, mas a dor ainda permanece sem resposta satisfatória. Esse tema continua incomodando muitos profissionais da saúde, estimulando-os a buscar respostas nas pesquisas, para melhor avaliação e seu controle.

A Enfermagem tem buscado estudar e contribuir para a ampliação dos conhecimentos sobre o tema. Em 1990, a Oncology Nursing Society adotou uma posição sobre a dor do paciente oncológico, definindo objetivos para a prática, considerações éticas e recomendações ${ }^{(2)}$.

No planejamento assistencial de enfermagem para suprir as necessidades fisiológicas e psicossociais do PODC, recomendado pela sociedade citada, destaca-se a necessidade dos enfermeiros contribuírem para o avanço da assistência ao paciente oncológico com dor crônica.

O envolvimento dos enfermeiros nas pesquisas sobre dor crônica e as terapêuticas, com a participação de médicos e pesquisadores de outras áreas do saber, é altamente significativo para o desenvolvimento de conhecimentos e de estratégias inovadoras para o cuidado do paciente. Para a autora, a forma como os profissionais de saúde atuam, comunicam-se entre si e como lidam com a dor do paciente são aspectos influenciados por suas definições ${ }^{(3)}$.

Porém, os tratamentos inadequados da dor crônica e dos outros sintomas do câncer geram problemas sérios, são negligenciados pelos profissionais de saúde; e todo paciente com câncer deveria obter o controle da sua dor como parte do seu cuidado ${ }^{(7)}$.

Portanto, o enfermeiro deve exercer seu papel no controle da dor, tem responsabilidade na avaliação diagnóstica, na intervenção e monitorização dos resultados do tratamento, na comunicação das informações da dor do paciente, como membro da equipe de saúde.

Considerar esse conjunto de fatores que interagem nos processos da dor crônica no paciente oncológico é um passo importante no sentido de avançar nas apropriações do objeto deste trabalho: a interpretação do cuidado pelos enfermeiros, protagonistas nesse cenário.

\section{PERCURSO METODOLÓGICO}

O estudo foi desenvolvido em uma instituição hospitalar governamental do interior do Estado do Paraná, onde a maioria dos pacientes internados é oncológico.

O projeto foi encaminhado e aprovado por um Comitê de Ética em Pesquisa.
Os informantes foram 8 enfermeiros com experiências no cuidado do PODC que aceitaram participar, assinando o termo de consentimento livre e esclarecido. Esses profissionais têm idades entre 27 e 45 anos; 3 são enfermeiros de 1 a 4 anos e 5 de 8 a 13 anos. Nenhum deles participou de um curso específico sobre oncologia ou dor.

A coleta de dados foi realizada pela entrevista semiestruturada que foi gravada com consentimento. Essas entrevistas foram conduzidas pelas questões: como você percebe a dor crônica no paciente oncológico?; como é cuidar do paciente oncológico com dor crônica?; como você se sente cuidando desse paciente?

Os dados foram transcritos na íntegra e a análise foi realizada seguindo as diretrizes do método qualitativo ${ }^{(8)}$ : ordenação, classificação em categorias empíricas e síntese e interpretação dos dados, com a descrição de categorias analíticas. A interpretação foi baseada na literatura e na nossa experiência profissional.

\section{RESULTADOS E DISCUSSÃO}

As categorias empíricas foram nomeadas como: humanização no atendimento, crítica ao modelo vigente, reação do paciente, cuidado, qualificação da equipe, doença, caracterização do paciente e dificuldades do cuidado.

Essas foram agrupadas em categorias analíticas que forneceram a nossa interpretação do cuidado do PODC, segundo a ótica dos enfermeiros. Foram identificadas três categorias analíticas: avaliação da dor; a importância do cuidado multidisciplinar e as dificuldades do cuidado, que serão apresentadas e discutidas a seguir.

A avaliação da dor do PODC

As descrições da dor crônicas do paciente oncológico, pelos enfermeiros, seguem os critérios do seu conhecimento e de suas crenças e valores. Os critérios que mais sobressaíram nos dados estão relacionados às alterações fisiológicas, aos comportamentos sociais e as atitudes apresentadas pelos pacientes, exemplificados nas falas:

Eu percebo a dor pela coloração da pele...

... pela expressão dele, pelo comportamento de rebeldia, por não aceitar você próximo a ele e ele rejeitar o tratamento.

Eu percebo a dor crônica por suas atitudes: deixa de alimentarse, fica depressivo e apático.

Vale ressaltar que o critério tomado com base para a avaliação da dor crônica, que emergiu com maior frequêencia entre todos os informantes, refere-se aos comportamentos e atitudes psicossociais do paciente, como: depressão, apatia, rebeldia, rejeição à situação e isolamento. 
Esses comportamentos e atitudes são denominados como comportamentos de dor $^{(9)}$. Quando alguém sente dor, os profissionais tomam conhecimento do fato porque o paciente demonstra o seu sofrimento por um comportamento. Tal comportamento, que é normal, serve para comunicar o que está acontecendo e solicitar apoio.

Outro aspecto que sobressaiu nas falas dos informantes, coerente com a exposição anterior, foi à relação da dor crônica com o sofrimento:

A gente percebe a dor crônica no olhar do paciente. É um paciente apático. Só pela fisionomia dele você já percebe que ele está com dor. Parece que ele transmite com o olhar, pedindo socorro: por favor, faça alguma coisa para aliviar a minha dor!

... é uma mistura de dor e raiva, incompreensão ...

... para ele é uma punição!

Por conseqüência, após valorizarem os comportamentos do paciente, os informantes expuseram a sua visão desse tipo de dor:

Eu vejo como uma das piores dores; os pacientes tornam-se dependentes das drogas e existe a dor psicológica, a não aceitação da doença, o abandono da família e amigos.

Os critérios utilizados pelos enfermeiros para avaliarem a dor crônica do paciente oncológico demonstram que eles baseiamse na sua empatia com o paciente, nas respostas emocionais e sociais dos pacientes, porém, também explicitam noções insuficientes sobre o tema. As suas impressões estão fundamentadas na cultura popular sobre a dor, determinada por suas crenças e valores. Assim, esse processo não é sistemático, não há utilização de protocolos, é essencialmente subjetivo e inadequado, incoerente com o conhecimento científico que deve fundamentar a prática profissional.

A avaliação da dor é um importante passo para 0 planejamento do cuidado. Ela impõe não apenas a determinação do problema físico do paciente, mas também os elementos psicológicos, sociais e emocionais do seu sofrimento e deve ser realizada, em conjunto, por todos os profissionais que acompanham o paciente.

A literatura apresenta diferentes protocolos de avaliação já desenvolvidos e avaliados, como o proposto pelo Instituto Nacional do Câncer - INCA, divulgado em 1997, que compreende todos os elementos fundamentais da avaliação ${ }^{(10)}$.

É preciso ressaltar que a avaliação da experiência dolorosa não é um procedimento simples, visto tratar-se de fenômeno individual e subjetivo, cuja interpretação e expressão envolvem elementos sensitivos, emocionais e culturais. Os objetivos da avaliação são caracterizar a experiência dolorosa em todos os seus domínios, identificar os aspectos que possam estar determinando ou contribuindo para manifestação dos sintomas, aferir as repercussões da dor no funcionamento biológico, emocional e social do indivíduo. Acredita-se que o insuficiente controle da dor crônica deva-se à avaliação inadequada ${ }^{(1)}$.
As crenças dos enfermeiros de que a dor crônica é às vezes sem solução, que a medicação não faz efeito e que os pacientes ficam dependentes das drogas, explicitam desinformações importantes a serem consideradas.

A terapêutica da dor no paciente oncológico tem como objetivo: aumentar as horas livres de dor, aliviar a dor durante 0 repouso do paciente, aliviar a dor que o paciente sente quando em posição ereta ou em atividade ${ }^{(10)}$.

0 alívio da dor crônica no paciente oncológico é possivel pela combinação medicamentosa de analgésicos opiáceos e não opiáceos. A utilização de analgésicos opiáceos associa-se ao desenvolvimento de dependência física e de tolerância, quando administrados de forma inadequada. Porém, a preocupação injustificada com a dependência psicológica tem levado os profissionais de saúde à utilização de doses inadequadas dessas drogas. A experiência clínica tem demonstrado que os pacientes oncológicos que recebem opiáceos com a finalidade de analgesia não se viciam, e isto é válido para adultos e crianças ${ }^{(10)}$.

$\mathrm{Na}$ relação com o PODC, os enfermeiros também expressam suas dificuldades em lidar com o sofrimento causado pelo câncer e pela dor.

Assim, a ênfase na descrição das respostas comportamentais, das atitudes, do sofrimento do paciente, da crença sobre a não eficácia dos tratamentos e da possibilidade de dependência às drogas pelos pacientes, retrata os conhecimentos culturais dos enfermeiros adquiridos durante a sua formação pessoal e profissional. As habilidades cognitivas e afetivas dos enfermeiros para lidarem com o tema, estão baseadas em características simbólicas. Da mesma forma que o câncer e a dor crônica, na cultura popular, são vistos como condições que carregam significados de impacto negativo para o paciente e seus familiares. Desse modo, é compreensível que para os enfermeiros essas situações também sejam desafiadoras. Porém, o conhecimento já desenvolvido sobre os temas fornece diferentes possibilidades de ações de enfermagem, tanto nos aspectos físicos como emocionais.

A importância do cuidado multidisciplinar com o PODC

O enfoque multidisciplinar do cuidado com o PODC emergiu com freqüência nas entrevistas com todos os informantes.

0 primeiro aspecto do cuidado multidisciplinar refere às dificuldades do enfermeiro para lidar com as respostas comportamentais e emocionais do PODC. Para alguns informantes, é valorizado o conhecimento específico de outros profissionais de saúde, principalmente o psicólogo, que poderia atuar contribuindo para o alívio da dor crônica do paciente oncológico e do seu sofrimento: 
... não se trabalha a parte psicológica e espiritual!

O cuidado deveria ser planejado junto com a equipe médica, psicólogo, assistente social, enfermeiro, fisioterapeuta e auxiliar de enfermagem!

0 modo como alguns enfermeiros valorizam o cuidado multidisciplinar não está baseado na integração de conhecimentos específicos de outras disciplinas mas, sim, para suprir a sua insegurança em lidar especificamente com os comportamentos psicossociais do PODC:

Eu tenho muitas dificuldades para cuidar desse paciente por não estar preparado.

... a gente fica sem ação. Na verdade a gente usa apenas a medicação e algum conforto. Eu acho que deveria haver um trabalho de psicologia em cima deles.

A natureza e a complexidade do cuidado do PODC requer que diferentes profissionais atuem de modo a favorecer a sua reabilitação física e psicossocial.

A atuação da equipe interdisciplinar tem como desafio proporcionar ao paciente uma experiência com outra gama de emoções, tais como: 0 amor, o alívio, a serenidade e a alegria ${ }^{(11)}$. A interdisciplinaridade do cuidado à saúde, surge como possibilidade de novas alianças, difíceis, mas que trará criatividade e avanço para o tratamento da dor crônica do paciente oncológico ${ }^{(12)}$.

Essa abordagem, que surgiu na década de 80 , é empregada para reduzir a dor por meio de modalidades terapêuticas fisiológicas, emocionais, cognitivas e sociais, para que o cuidado seja resgatado com qualidade ${ }^{(2)}$.

As dificuldades do cuidado do PODC

Nessa categoria, apresentamos como o cuidado do PODC é compreendido pelos enfermeiros.

Para um dos informantes, o cuidado desse paciente é semelhante ao dos demais:

O paciente oncológico com dor crônica ou qualquer outra clínica ... o cuidado deve ser integral!

Para os outros enfermeiros entrevistados, o cuidado com o PODC é diferente:

Eu o vejo de uma forma diferente dos demais pacientes. É um paciente que precisa de um cuidado mais direto da equipe e da familia,... a gente tem que ter um cuidado especial. Ele já é um paciente sofrido...

0 modo como os enfermeiros reconhecem o cuidado do PODC, ressaltando a necessidade da presença do psicólogo neste cuidado, reforçam as suas dificuldades dos enfermeiros para lidarem com esse paciente.

Entretanto, o enfermeiro tem empatia com o sofrimento do PODC e enaltece o cuidado humanizado:
Esse cuidado humanizado é muito mais que assistência, mais que administrar drogas e achar que tudo foi feito. 0 cuidado é estar atento as intercorrências e observar as atividades dos pacientes. Não deixá-lo sozinho, tratar como ser humano, tentar amenizar o sofrimento através de medicamentos, conforto físico e apoio psicológico (...) apaziguando a sua revolta.

Embora haja o discurso da importância da empatia e do cuidado humanizado com o PODC, frente às suas dificuldades para desenvolve-lo, a presença do psicólogo seria uma "tábua de salvação" para adequar o cuidado. Neste contexto, as necessidades psicológicas do paciente não são supridas e suas ações abordam apenas a dimensão física:

Eu tento amenizar o sofrimento com medicamentos, conforto físico...

Na realidade, eu não tenho estrutura e nem conhecimento para cuidar do PODC. O que faço é administrar analgésicos que geralmente são paliativos..

O cuidado humanizado emerge com freqüência nas falas dos enfermeiros e é uma abordagem da enfermagem pautada em pressupostos filosóficos humanísticos que tem acompanhado a profissão, nas últimas décadas, ainda como característica ideológica ${ }^{(13)}$.

Os enfermeiros descrevem que suas ações são limitadas pela falta de conhecimento de como intervir na dimensão afetiva ou expressiva. Assim, o cuidado prestado é baseado no modelo médico, no qual os profissionais têm maior segurança.

Os profissionais de saúde não médicos e o público em geral não aceitam este modelo médico, pois, para eles, o câncer é considerado como uma doença com repercussões sociais e psicológicas e não apenas como uma doença estabelecida em um órgão. Portanto, busca-se a adoção de uma medicina mais humana, guiada pela alta tecnologia, pesquisa e integração de disciplinas, para a promoção da qualidade de vida do paciente. Nesse sentido, a disciplina de psico-oncologia tem muita a oferecer para a enfermagem, pois ela apresenta pressupostos que abordam a relação fisiológica e psicossocial do câncer, para o indivíduo e sua família ${ }^{(11)}$.

$\mathrm{Na}$ maioria das falas dos informantes está explícita a dificuldade para agir com o PODC, por não existir uma equipe especializada, para lidar com esse perfil de indivíduos; a falta de profissionais capacitados; por não existir uma equipe estruturada; e não haver protocolos estabelecidos. As declarações dos informantes sinalizam, também, que a falta desse conjunto de condições, remeteos a uma prática tarefeira que acaba negligenciando o cuidado humanizado.

Cuidar envolve atos humanos no processo de assistir ao indivíduo, ao grupo ou à comunidade, os quais são deliberados, racionais, dotados de sentimento e fundamentados em conhecimento $^{(14)}$. 
Os resultados deste estudo levam-nos a perspectiva de que a educação continuada é necessária para eliminar a ignorância disseminada dos profissionais de saúde sobre o câncer, a dor crônica e o controle da dor no câncer, pois, a atenção deve ser dada a todos os aspectos do sofrimento - físico, psicológico, social e espiritual ${ }^{(15)}$.

\section{CONSIDERAÇÕES FINAIS}

A dor crônica no paciente oncológico é considerada como problema de saúde pública nos EUA e no Brasil, pois milhões de dólares e reais são gastos com internações hospitalares de pacientes sem que haja melhoria em sua qualidade de vida ${ }^{\left({ }^{(10)}\right.}$. Os resultados desse estudo confirmam o que autores nacionais e internacionais consideram que um dos grandes problemas a ser enfrentado pelos sistemas de saúde dos países, relaciona-se com a capacitação dos profissionais para cuidarem do PODC.

As desinformações dos enfermeiros deste estudo, sobre os resultados dos métodos de controle da dor crônica, devido a inadequação da sua formação, os levam a: não avaliarem a dor de forma sistemática; a subestimarem a freqüência de ocorrência da dor e a ignorarem o impacto devastador da dor para o indivíduo. Desse modo, os informantes comportam-se como se a dor fizesse parte da doença e do tratamento, como um sintoma natural.

\section{REFERÊNCIAS BIBLIOGRÁFICAS}

1. Pimenta CAM, Koizumi MS, Teixeira MJ. Dor no doente com câncer: características e controle. Rev Bras Cancerol 1997 jan/fev/março; 43(1):21-44.

2. Cailliet R. Dor: mecanismos e tratamento. Porto Alegre (RS): Artes Médicas; 1999.

3. Benoliel JQ. Multiple meaning of pain and complexities of pain management. Nurs Clin North Am 1995 December; 30(4):583-96.

4. Teixeira MJ, Shibata MK, Pimenta CAM, Corrêa CF. Dor no Brasil: estado atual e perspectivas. São Paulo(SP): Limay; 1995.

5. Lobato O. O problema da dor. In: Mello J Filho, organizador. Psicossomática hoje. Porto Alegre (RS): Artes Médicas; 1992. p.16578.

6. Smeltzer SC, Bare BG. Brunner \& Suddarth: tratado de enfermagem médico-cirúrgica. $8^{a}$ ed. Rio de Janeiro (RJ): Guanabara Koogan; 1998.

7. Johansson FG, Dickson JMF. The importance of nursing research design and methods in cancer pain management. Nurs Clin North Am 1995 December; 30(4):597-607.

8. Minayo MCS. O desafio do conhecimento: pesquisa qualitativa em saúde. $4^{\mathrm{a}}$ ed. São Paulo (SP): Hucitec; 1998.
Os resultados também mostram que o cuidado humanizado é mais uma prática discursiva do que uma ação objetiva. Os enfermeiros fundamentam suas ações no modelo médico e que são ineficazes.

Para cuidar do paciente oncológico é preciso: conhecer, saber 0 que 0 outro necessita e como podemos ajudá-lo nesse processo $^{(14)}$.

A incerteza, a tensão do trabalho a importância e a percepção do peso da tarefa de cuidar do PODC produz um desconforto e uma baixa auto-estima nos enfermeiros. Daí a necessidade deles aprimorarem seus conhecimentos e habilidades para poderem com segurança e eficiência cuidar do PODC e sua família.

Vale insistir que não se deve subestimar ou mesmo ignorar as dimensões éticas, culturais, históricas e religiosas envolvidas na temática. Hoje em oncologia, a preocupação não é mais somente com a cura mas, sim, com a qualidade de vida do paciente.

Devido à magnitude do problema do cuidado com o PODC, expostos neste estudo, faz-se necessárias intervenções que venham transformar esse cuidado. Como estratégia, consideramos que o currículo de graduação de enfermeiros deva inserir conteúdos sobre o tema. Em nível institucional, a educação continuada é a recomendação para se alcançar às metas de qualidade do cuidado com o PODC.

9. Capisano HF. Imagem Corporal. In: Mello J Filho, organizador. Psicossomática hoje. Porto Alegre (RS): Artes Médicas; 1992. p.17992.

10. Instituto Nacional do Câncer (INCA). 0 alívio da dor do câncer. $2^{a}$ ed. Rio de Janeiro (RJ): INCA; 1997.

11. Schulze CMN. Dimensões da dor no câncer: reflexões sobre o cuidado interdisciplinar e um novo paradigma de saúde. São Paulo (SP): Robe; 1997.

12. Canesqui AM. Organização: dilema e desafios das ciências sociais na Saúde Coletiva. São Paulo (SP); 1995.

13. Vieira MJ. A representação do cuidar na imagem cultural da enfermagem. Rev Latino-am Enfermagem 1999 dezembro; 7(5):2531.

14. Arruda EM, Marcelino SR. Cuidando e confortando. In: Schulze CMN. Dimensões da dor no câncer: reflexões sobre o cuidado interdisciplinar e um novo paradigma de saúde. São Paulo (SP): Editora Robe; 1997. p. 157-89.

15. Twycross RG. Alívio da dor no câncer. In: Schulze CMN. Dimensões da dor no câncer: reflexões sobre o cuidado interdisciplinar e um novo paradigma para a saúde. São Paulo (SP): Robe; 1997. p. 83-115. 